\title{
Venous thromboembolism risk and prophylaxis prescription in surgical patients at a tertiary hospital in Eastern Cape Province, South Africa
}

\author{
W D Rocher, ${ }^{1} \mathrm{MB}$ ChB; T Page, ${ }^{1} \mathrm{MB}$ ChB; M Rocher, ${ }^{1} \mathrm{MB}$ ChB; D Nel, ${ }^{2} \mathrm{MB}$ ChB \\ ${ }^{1}$ Department of Surgery, Frere Hospital, East London Hospital Complex, South Africa \\ ${ }^{2}$ Department of General Surgery, Faculty of Health Sciences, Groote Schuur Hospital and University of Cape Town, South Africa
}

Corresponding author: W D Rocher (wilhelmusrocher@gmail.com)

\begin{abstract}
Background. Venous thromboembolism (VTE) is a common complication during and after hospitalisation, and is regarded as the most common cause of preventable death in hospitalised patients worldwide. Despite its importance, there are few data on VTE risk and adherence to prophylaxis prescription guidelines in surgical patients from the South African (SA) public sector, especially from lowresource environments such as Eastern Cape Province.

Objectives. To evaluate the risk and prescription of VTE prophylaxis to surgical patients at a tertiary government hospital in the Eastern Cape. Methods. A cross-sectional clinical audit of general surgical inpatients was performed on two dates during July and August 2017. Patients' VTE risk was calculated by using the Caprini risk assessment model (RAM) and thromboprophylaxis prescription evaluated accordingly. Results. A total of 179 patients were included in the study, of whom 56\% were male and $44 \%$ female. The average age was 45 (range 18 83 ) years. Of the total number of participants, $33 \%$ were elective cases and $67 \%$ were emergency admissions. With application of RAM, $77 \%$ of patients were at risk of VTE (Caprini score $\geq 2$ ), with $81 \%$ of elective and $74 \%$ of emergency patients being at risk. The most prevalent risk factors for VTE were major surgery (34\%), age 41 - 60 years (30\%), age 61 - 74 years (20\%) and sepsis during the previous month (27\%). A contraindication to chemoprophylaxis was recorded in $30 \%$ of patients, with the most prevalent being renal dysfunction (40\%), peptic ulcer disease (34\%), active bleeding (17\%), liver dysfunction (17\%), coagulopathy (6\%) and recent cerebral haemorrhage (6\%). With regard to VTE risk profile and contraindications to chemoprophylaxis, the correct thromboprophylactic treatment was prescribed to $26 \%$ of at-risk patients, with $21 \%$ of elective and $27 \%$ of emergency admission patients receiving the correct therapy. Conclusions. Despite a high proportion of patients being at risk of VTE, the rate of adequate thromboprophylaxis prescription for surgical inpatients at Frere Hospital, East London, SA is very low. Increased availability of mechanical prophylaxis, as well as interventions to improve the rate of adequate prophylaxis prescription, needs to be evaluated for feasibility and effect in this hospital and other SA public hospitals.
\end{abstract}

S Afr Med J 2019;109(3):178-181. DOI:10.7196/SAMJ.2019.v109i3.13510

Venous thromboembolism (VTE) is a common complication during and after hospitalisation for acute medical illness and surgery. Untreated proximal deep vein thrombosis (DVT) is associated with a $30-50 \%$ rate of pulmonary embolism (PE), which is regarded as the most common cause of preventable death in hospitalised patients, contributing to $10 \%$ of all in-hospital deaths. ${ }^{[1-4]}$ In addition to the acute risk of mortality, VTE is also associated with the long-term risk of post-thrombotic syndrome and post-thrombotic pulmonary hypertension. The multinational ENDORSE (Epidemiologic International Day for the Evaluation of Patients at Risk for Venous Thromboembolism in the Acute Hospital Care Setting) study showed that more than half of hospitalised patients are at risk of VTE and that surgical patients have a higher risk (64\%) than medical patients $(41.5 \%){ }^{[1]}$ In terms of local data, the second wave of the Gauteng Province-based TUNE-IN (The Use of VTE prophylaxis in relatioN to patiEnt risk profiling) study included 453 inpatients from multiple surgical specialties, a third of whom were from the state sector, and found that $92 \%$ were at risk of VTE. ${ }^{[5]}$

In 2007, the National Institute for Health and Care Excellence (NICE) recommended that all patients admitted to hospital should undergo a formal VTE risk assessment, and that appropriate prophylaxis should be prescribed. ${ }^{[6]}$ In 2012, the American College of Chest Physicians (ACCP) published antithrombotic therapy and prevention of thrombosis guidelines, which uses the Caprini risk assessment model (RAM) to assess the degree of VTE risk. ${ }^{[7]}$ SA guidelines were published in 2013, which closely mirror the previous ACCP guidelines. ${ }^{[4,8]}$ Despite the unfavourable figures and formalised recommendations, thromboprophylaxis is found to be under-prescribed. Worldwide data suggest that $>50 \%$ of hospitalised patients are at risk of DVT, with only half of these receiving an ACCPrecommended method of prophylaxis. ${ }^{[7,9]}$

SA public sector data on VTE risk and adherence to prophylaxis prescription guidelines are lacking, especially from provinces such as the Eastern Cape, where there is a lack of resources and infrastructure. ${ }^{[10,11]}$ The objective of this study was therefore to evaluate the VTE risk and prescription of adequate prophylaxis to surgical patients at a tertiary government hospital in Eastern Cape Province.

\section{Methods}

A cross-sectional audit of general surgical inpatients was performed on two dates during July and August 2017. The two dates were 30 days apart to avoid overlap of patients with a prolonged admission. The 
timing of the study and its objectives were at no stage discussed with treating surgeons or ward staff to avoid affecting prescription behaviour.

Inclusion criteria were adult patients admitted to general surgical wards in the Department of General Surgery, comprising elective and emergency admissions, the latter including trauma and burn patients. Those $<18$ years of age or those who were treated in the intensive care unit (ICU) were not included. Patients who refused consent to participate in the study were excluded.

For each patient, individual risk factors for VTE were recorded and the overall VTE risk score was calculated using the Caprini RAM (Table 1), with the final VTE risk categorised as low, moderate, high and highest (Table 2). Medication charts were reviewed to assess for current thromboprophylaxis therapy and compared with therapy suggested by the Caprini RAM and ACCP guidelines. ${ }^{[2,7]}$ Contraindications to thromboprophylaxis, as mentioned in the ENDORSE and AVAIL-ME (Assessment for VTE Management in Hospital - Middle East) studies, were also recorded. ${ }^{[1,9]}$ A patient with a VTE score of $\geq 2$ was considered 'at risk' and regarded as receiving correct therapy if the prophylactic treatment met the minimum therapy recommended for their risk category. Patients who did not receive the minimum therapy required for their risk category, or had clearly documented contraindications to chemoprophylaxis but nonetheless received it, were considered to be administered the incorrect therapy.

\section{Ethical approval}

Ethical approval for this study was granted by the East London Hospital Complex (affiliated with Walter Sisulu University) ethics review board.

\section{Results}

\section{Demographics}

Of 181 eligible patients, 179 were included in the study - in 2 cases consent could not be obtained. Of those included, $56 \%$ were male and $44 \%$ female. The average age of patients was 45 (range $18-83$ ) years. There were 59 elective surgical admissions and 120 emergency admissions. Emergency admissions comprised 80 acute surgical, 23 trauma and 17 burn patients.

\section{Risk assessment}

With application of RAM, $77 \%$ of patients were at risk of DVT (Caprini score $\geq 2$ ), with $81 \%$ of elective and $74 \%$ of emergency admissions being at risk. The most prevalent risk factors for VTE

\begin{tabular}{|c|c|c|c|c|}
\hline $\begin{array}{l}\text { Each of the following risk } \\
\text { factors represents } 1 \text { point }\end{array}$ & $\begin{array}{l}\text { Each of the following } \\
\text { risk factors represents } \\
2 \text { points }\end{array}$ & $\begin{array}{l}\text { Each of the following risk } \\
\text { factors represents } \\
3 \text { points }\end{array}$ & $\begin{array}{l}\text { Each of the following } \\
\text { risk factors represents } \\
5 \text { points }\end{array}$ & $\begin{array}{l}\text { For women only ( } 1 \text { point } \\
\text { each) }\end{array}$ \\
\hline Age $41-60$ years & Age 60 - 74 years & Age $\geq 75$ years & Elective major lower- & Oral contraception or HRT \\
\hline Minor surgery planned & Major surgery (>45 min) & History of SVT, DVT/PE & extremity arthroplasty & Pregnancy or postpartum \\
\hline History of prior major & Arthroscopic surgery & Family history of DVT/PE & Hip, pelvis or leg fracture & Unexplained stillbirth, \\
\hline surgery $(<1 \mathrm{mo})$ & Laparoscopic surgery & Congenital thrombophilia & $(<1 \mathrm{mo})$ & miscarriage (>3), SGA \\
\hline Varicose veins & $(>45 \mathrm{~min})$ & Positive factor V Leiden & Stroke $(<1 \mathrm{mo})$ & \\
\hline History of inflammatory & Previous and current & Positive prothrombin & Multiple trauma (<1 mo) & \\
\hline bowel disease & malignancy & $20210 \mathrm{~A}$ & Acute spinal cord injury & \\
\hline Swollen legs (current) & Morbid obesity (BMI & Elevated serum homo- & (paralysis) $(<1 \mathrm{mo})$ & \\
\hline Obese $($ BMI $>25)$ & $\left.>40 \mathrm{~kg} / \mathrm{m}^{2}\right)$ & cysteine & & \\
\hline Acute myocardial infarction & Patient confined to bed & Acquired thrombophilia & & \\
\hline Congestive heart failure & $(>72 \mathrm{~h})$ & Positive lupus anti- & & \\
\hline$(<1 \mathrm{mo})$ & Immobilising plaster cast & coagulant & & \\
\hline Sepsis $(<1 \mathrm{mo})$ & $(>72 \mathrm{~h})$ & Elevated anticardiolipin & & \\
\hline Abnormal pulmonary & Central venous access & antibodies & & \\
\hline function (COPD) & & Heparin-induced & & \\
\hline Medical patient (current bed & & thrombocytopenia & & \\
\hline \multicolumn{5}{|l|}{ rest) } \\
\hline \multicolumn{5}{|l|}{ Other risk factors (excluding } \\
\hline diabetes) & & & & \\
\hline
\end{tabular}

Table 2. Caprini risk assessment model categories and recommended prophylaxis ${ }^{[2,8,12]}$

\begin{tabular}{llll}
\hline $\begin{array}{l}\text { Risk factor } \\
\text { score, total }\end{array}$ & Incidence of DVT, \% & Risk level & Prophylaxis regimen \\
\hline $0-1$ & $<10$ & Low & No specific measures, early ambulation \\
2 & $10-20$ & Moderate & ES/GCS, IPC, LDUH or LMWH \\
$3-4$ & $20-40$ & High & IPC, LDUH or LMWH \\
$\geq 5$ & $40-60$ & Highest & Pharmacological: LDUH, LMWH, warfarin or F-Xa inhibitor alone \\
& $(1-5$ mortality) & & or in combination with ES/GCS or IPC
\end{tabular}

DVT $=$ deep vein thrombosis; ES/GCS = elastic stockings/graduated compression stockings; IPC $=$ intermittent pneumatic compression; LDUH $=$ low-dose unfractionated heparin; $\mathrm{LMWH}=$ low-molecular-weight heparin; F-Xa = factor Xa. 
were major surgery (34\%), age $41-60$ years (30\%), age $61-74$ years (20\%) and sepsis during the previous month (27\%). Other notable risk factors were minor surgery (16\%), malignancy (9\%), patient being bed bound for $>72$ hours (7\%), and a history of major surgery within the previous month $(6 \%)$.

\section{Contraindications to chemoprophylaxis}

Of the total number of patients included in the study, a contraindication to chemoprophylaxis was recorded in 53 (30\%). The most common contraindications were: renal dysfunction (40\%), peptic ulcer disease (34\%), active bleeding (17\%), liver dysfunction (17\%), coagulopathy (6\%) and recent cerebral haemorrhage (6\%). Of these 53 patients, 34 (19\% of total patients) were also at risk of VTE (Caprini score $\geq 2$ ), necessitating mechanical prophylaxis. Despite a clear contraindication, 10 of the 34 patients received some form of chemoprophylaxis.

\section{Therapy}

Of the total number of at risk patients, $26 \%$ received the correct thromboprophylactic treatment according to their risk factor profile, while $21 \%$ of elective patients and $27 \%$ of emergency patients received the correct therapy. The number of patients in each risk group and the rate of correct prescription of prophylaxis are shown in Table 3.

\section{Discussion}

VTE is a common complication during and after hospitalisation and is considered the most common preventable cause of inhospital mortality worldwide. Despite its significance, there is a paucity of data to describe the VTE risk and prophylaxis prescription in SA surgical patients, especially in the public sector. This study therefore aimed to describe the VTE risk and prophylaxis prescription practices at a tertiary public hospital in the Eastern Cape.

With application of the Caprini RAM, 77\% of patients were found to be at risk of DVT. This figure is higher than the average of $64 \%$ in the ENDORSE study, but within the range of $44-80 \%$ for the 32 participating nations. ${ }^{[1]}$ In terms of prophylaxis prescription, $26 \%$ of patients in the current study were found to be receiving the correct thromboprophylaxis according to their risk factor profile. The rate of adequate VTE prophylaxis is therefore much lower than that in the ENDORSE study, where the overall correct rate of prophylaxis prescription in surgical patients was $59 \% \cdot{ }^{[1]}$

The low correct prophylaxis prescription rate may be owing to the initial assessment and management of patients in this hospital setting usually being done after hours by junior doctors (interns), who might not possess the experience to gauge VTE risk clinically or be confident to prescribe anticoagulation medication safely. Considering that emergency patient admissions formed a large portion of the sample (67\%), of whom most would have been admitted after hours by intern doctors, this may be a significant factor. The high burden of acute surgical, trauma and burn cases in many secondary and tertiary hospitals in SA, indicates that reliance on junior doctors to manage

$\begin{aligned} & \text { Table 3. Risk stratification and prophylaxis prescription for } \\
& \text { at-risk surgical admissions }\end{aligned}$
\begin{tabular}{|lll}
\hline Caprini VTE risk score & Patients, $\boldsymbol{n}$ & Correct treatment, $\boldsymbol{n}(\%)$ \\
\hline Moderate (2) & 36 & $4(12)$ \\
High $(3-4)$ & 58 & $16(28)$ \\
Highest $(\geq 5)$ & 43 & $15(35)$ \\
Total & 137 & $35(26)$ \\
VTE = venous thromboembolism. & &
\end{tabular}

patients after hours is unlikely to be unique to this hospital. Although after-hours admissions are reviewed during a post-intake consultantled ward round the next day, time spent with each patient is limited and thus the medication charts may not be fully reviewed for each patient during a busy and problem-focused round. For resourcelimited settings, such as the public sector in the Eastern Cape where many senior posts are unfilled, daily consultant-led ward rounds are often not feasible; hence, greater responsibility falls upon the shoulders of junior doctors, who may lack the experience to evaluate ongoing VTE risk once the patient is admitted.

VTE risk for elective and emergency admissions was fairly similar - $81 \%$ and $74 \%$, respectively. However, in terms of prophylaxis, the correct therapy was prescribed to only $21 \%$ of elective patients compared with $27 \%$ of emergency patients. Although VTE is a problem in all patients, it is an enormous problem in patients admitted for an elective procedure/work-up, where a favourable outcome is usually anticipated, especially when a catastrophic event such as fatal PE occurs. Although elective patients are admitted from the surgical outpatient department, where there is usually consultant supervision, the responsibility again primarily falls upon the interns to clerk and admit these patients, including their ward prescription charts.

Another factor contributing to poor adherence to prophylaxis guidelines was the lack of availability of mechanical prophylaxis in the surgical wards at Frere Hospital. Therefore, a patient with a Caprini score of $\geq 2$, who also had a clear contraindication to chemoprophylaxis, was automatically regarded as receiving the incorrect therapy. Given that $19 \%$ of all patients included in the study needed mechanical prophylaxis, the lack thereof points to an institutional failure that is likely to be present at many other hospitals in similar resource-limited environments. Ten patients received chemoprophylaxis despite having a documented contraindication, which may point to the inexperience of prescribers to consider bleeding risk or to desperate clinicians who have to make judgement calls on prophylaxis for high VTE-risk patients outside of guideline recommendations, thus exposing their patients to potential harm. The current shortage of unfractionated heparin in SA, with it becoming a section 21 drug, has often affected access to the only appropriate pharmacological thromboprophylaxis available at many state hospitals. This highlights another example of a system failure that may influence prescriber compliance with appropriate therapy in this setting.

SA is a resource-limited environment, where many hospitals continue to rely heavily on inexperienced junior doctors to manage patients, especially after-hours admissions and ongoing routine ward care. Numerous interventions have been proposed to improve VTE prophylaxis prescription, such as electronic alert systems, an opt-out thromboprophylaxis policy, performance evaluation and feedback, as well as practitioner education. ${ }^{[13-17]}$ Although electronic prescription alerts and performance reviews are not be feasible in our setting, the inclusion of a risk assessment sheet on the inpatient prescription charts and providing continuous practitioner education are interventions that should be considered for improving VTE prophylaxis prescription. With such a high percentage of patients at risk, an opt-out policy of thromboprophylaxis prescription should also be considered. ${ }^{[18]}$ Increased availability of mechanical prophylaxis for patients with contraindications to chemoprophylaxis is also essential, as a lack thereof significantly hampers the clinician's ability to safely prevent VTE in patients with bleeding risks.

\section{Study limitations}

A limitation of this study was that data were collected at a single point in time, with patients already admitted to the surgical ward. As 
there is evidence that a 24-hour delay in initiating VTE prophylaxis is linked to increased mortality, it would have been interesting to know how many patients started receiving the correct therapy according to their risk category, but also started at the right time. ${ }^{[19]}$ This is especially relevant in the SA state sector, where patients may be left in busy admission units before being transferred to a ward.

Another limitation of this single-point-in-time study was that the total duration of thromboprophylaxis was not reviewed, which is a further important factor to evaluate if correct thromboprophylaxis had been given during and after a patient's hospital stay.

\section{Conclusion}

Despite a high proportion of patients at risk for VTE, the rate of adequate thromboprophylaxis prescription for surgical inpatients at Frere Hospital is very low in both emergency and elective groups. The lack of mechanical prophylaxis and heavy reliance on junior doctors for medication prescription may be factors contributing to the low compliance rate. More research is needed to assess the risk for and impact of this common cause of morbidity and mortality in similar populations. Moreover, interventions to improve the rate of adequate prophylaxis prescription need to be evaluated for feasibility and effect in SA public sector hospitals.

Declaration. None.

Acknowledgements. None.

Author contributions. The authors contributed equally to all aspects of the research and the writing of the article.

Funding. None.

Conflicts of interest. None.

1. Cohen AT, Topson VF, Bergmann JF, et al. Venous thromboembolism risk and prophylaxis in the acute hospital care setting (ENDORSE study): A multinational cross sectional study. Lancet 2008;371(9610):387-394. https://doi.org/10.1016/S0140-6736(08)60202-0
2. Bahl V, Hu HM, Henke PK, Wakefield TW, Campbell DA, Caprini JA. Validation study of a retrospective venous thromboembolism risk scoring method. Ann Surg 2009;30(20):1-7. https://doi. org/10.1097/SLA0b013e3181b7fca6

3. Laryea J, Champagne B. Venous thromboembolism prophylaxis. Clin Colon Rectal Surg 3. Laryea J, Champagne B. Venous thromboembolism p
2013;26(3):153-159. https://doi.org/10.1055/s-0033-1351130

4. Jacobson BF, Louw S, Buller H, et al. Venous thromboembolism: Prophylactic and therapeutic practice guideline. S Afr Med J 2013;103(4):260-267. https://doi.org/10.7196/SAMJ.6706

5. Jacobson BF, Louw S, Riback WJ. The use of VTE prophylaxis in relation to patient risk profiling (TUNE-IN) wave 2 study. S Afr Med J 2014;104(12):880-884. https://doi.org/10.7196/SAMJ.8456

6. Hill J, Treasure T. Reducing the risk of venous thromboembolism (deep vein thrombosis and pulmonary embolism) in inpatients having surgery: Summary of NICE guidance. BMJ 2007;334(7602):1053. https://doi.org/10.1136/bmj.39174.678032.ad

7. Gould MK, Garcia DA, Wren SM, et al. Antithrombotic therapy and prevention of thrombosis, 9th ed: American College of Chest Physicians evidence-based clinical practice guidelines. Chest 2012;141(2):227-277. https://doi.org/10.1378/chest.11-2297

8. Geerts WH, Bergqvist D, Pineo GF, et al. Prevention of venous thromboembolism: American College of Chest Physicians evidence based clinical practice guidelines (antithrombotic and thrombolytic of Chest Physicians evidence based clinical practice guidelines (antithrombotic and thrombolytic
therapy), 8th ed: ACCP Guidelines. Chest 2008;133:S381-S453. https://doi.org/10.1378/chest.08-0656

9. Therer AT, Aoun J, Salameh P. The AVAIL-ME study: A multinational survey of VTE risk and prophylaxis. J Thromb Thrombolys 2011;31(1):47-56. https://doi.org/10.1007/s11239-010-0492-2

10. Mayosi BM, Benatar SR. Health and health care in South Africa - 20 years after Mandela. N Engl J Med 2014;371(14):1344-1353. https://doi.org/ 10.1056/NEJMsr1405012

11. Treatment Action Campaign and Section 27 Catalysts for Social Justice. Death and dying in the Eastern Cape: An investigation into the collapse of a health system. http://section27.org.za/wp-content/ uploads/2014/10/SECTION27-report-redacted.pdf (accessed 1 March 2018).

12. Wessels PF, Riback WJ. DVT prophylaxis in relation to patient risk profiling - the TUNE-IN study. S Afr Med J 2012;102(2):85-89. https://doi.org/10.7196/SAMJ.4859

13. Cassidy MR, Rosenkranz P, McAneny D. Reducing postoperative venous thromboembolism complications with standardized risk-stratified prophylaxis protocol and mobilization programme. J Am Coll Surg 2014;218(6):1095-1104. https://doi.org/10.1016/j.jamcolsurg.2013.12.061

14. Kucher N, Koo S, Quiroz R, Cooper JM. Electronic alerts to prevent venous thromboembolism among hospitalized patients. N Engl J Med 2005;352(22):969-977. https://doi.org/10.1056/NEJMoa041533

15. Lau BD, Arnaoutakis GJ, Streiff MB, et al. Individualised performance feedback to surgical residents improves appropriate venous thromboembolism prophylaxis prescription and reduces potentially improves appropriate venous thromboembolism prophylaxis prescription and reduces potentially
preventable VTE: A prospective cohort study. Ann Surg 2015;20(10):1-7. https://doi.org/10.1097/ preventable VTE: A

16. Beutel BG, Jenkins LS. Preventing venous thromboembolism at a district hospital: A quality improvement study. S Afr Fam Pract 2015;57(2):64-68. https://doi.org/10.1080/20786190.2014. 977033

17. Kahn SR, Morrison DR, Cohen JM, et al. Interventions for implementation of thromboprohylaxis in hospitalized medical and surgical patients at risk for venous thromboembolism (review). Cochrane Database Syst Rev 2013;(7):1-186. https://doi.org/10.1002/14651858.CD008201.pub2

18. Anderson SB, Lin SN, Reiss J, Skupski D, Grunebaum A. Peripartum thromboprophylaxis before and after implementation of a uniform heparin protocol. J Perinat Med 2014;42(2):219-223. https://doi, org/10.1515/jpm-2013-0165

19. Ho KM, Chavan S, Pilcher D. Omission of early thromboprophylaxis and mortality in critically ill patients: A multicenter registry study. Chest 2011;140(6):1436-1446. https://doi.org/10.1378/chest.11-1444

Accepted 21 August 2018. 